\title{
Surto familiar de botulismo no Estado do Ceará: relato de caso
}

\author{
Familiar outbreak of botulism at Ceará State, Brazil: case report
}

\author{
Morgana Maria de Oliveira Barboza ${ }^{1}$, Norival Ferreira dos $\operatorname{Santos}^{2}$ e Oscarina Viana de Sousa ${ }^{3}$
}

\section{RESUMO}

Relato de surto familiar de botulismo por intoxicação alimentar, envolvendo um óbito, onde foram encontradas lacunas no preenchimento do prontuário. O objetivo foi descrever a patologia chamando a atenção dos profissionais de saúde para o fornecimento adequado de informações relevantes para a investigação epidemiológica de doenças de notificação compulsória.

Palavras-chaves: Botulismo alimentar. Surto familiar. Vigilância epidemiológica.

\begin{abstract}
Report of a family outbreak of botulism food poisoning involving a death, where gaps in the completion of medical records were identified. The study aimed to describe the pathology and emphasize to health professionals the need to provide adequate information relevant to epidemiological investigation of compulsory notification diseases.
\end{abstract}

Keywords: Botulism food. Family outbreak. Epidemiological surveillance.

\section{INTRODUÇÃO}

O termo botulismo é utilizado para designar a intoxicação pelo Clostridium botulinum. O nome provém da palavra botulus (salsicha) em latim, alimento envolvido nos primeiros casos de botulismo, cientificamente comprovados, ocorridos na Europa Central no fim do século XVI.

O botulismo é uma enfermidade resultante da ação de potente neurotoxina de origem proteica produzida pelo Clostridium botulinum, que bloqueia a neurotransmissão em sinapses colinérgicas, causando paralisia simétrica descendente. A doença tem distribuição mundial e acomete pessoas em casos isolados ou causa surtos familiares. É considerado um problema de saúde pública devido a sua gravidade e alta letalidade. De ocorrência súbita, caracteriza-se por manifestações neurológicas seletivas, de evolução dramática e elevada mortalidade.

O Center for Diasease Control and Prevention (CDC) $)^{1}$ classifica o botulismo em quatro categorias epidemiológicas: $1^{\mathrm{a}}$ ) botulismo por intoxicação alimentar; $2^{a}$ ) botulismo infantil; $3^{a}$ ) botulismo por lesão e 4a) botulismo indeterminado ou por colonização intestinal em adultos. Este último é menos comum, sendo semelhante ao botulismo

1. Laboratório de Bioquímica Humana, Setor de Microbiologia, Universidade Estadual do Ceará, Fortaleza, CE. 2. Escola de Saúde Pública, Secretaria de Saúde do Estado do Ceará, Fortaleza, CE. 3. Laboratório de Microbiologia Ambiental e do Pescado, Instituto de Ciências do Mar, Universidade Federal do Ceará, Fortaleza, CE.

Endereço para correspondência: Dra. Oscarina Viana de Sousa. Labomar/UFC. Av. Abolição 3207, Meireles, 60165-081 Fortaleza, CE.

Tel: 5585 3366-7027

e-mail: oscarinasousa@yahoo.com.br

Recebido para publicação em 12/08/2010

Aceito em 04/02/2011 infantil, mas atingindo crianças maiores de um ano de idade e adultos. O botulismo alimentar ocorre pela ingestão da toxina pré-formada, enquanto que nos outros três tipos a enfermidade ocorre pela infecção, multiplicação e produção de toxinas por microrganismos clostridiais em feridas ou no trato gastrintestinal.

Atualmente, são descritos oito tipos de toxinas botulínicas: A, $\mathrm{B}, \mathrm{C}_{1}, \mathrm{C}_{2}, \mathrm{D}, \mathrm{E}, \mathrm{F}$ e $\mathrm{G}$, que se distinguem por suas características antigênicas. Entre elas, os tipos A, B, E e, mais raramente, F são causas de doenças nos seres humanos. O tipo $G$ é pouco conhecido e foi associado a alguns casos de morte súbita² ${ }^{2}$.

As cepas de C. botulinum são classificadas em quatro grupos de acordo com o tipo de toxina que produzem. O grupo I inclui todas as cepas produtoras do tipo A e dos tipos B e F proteolíticas; pertencem ao grupo II todas as cepas com toxina do tipo $\mathrm{E}$ e aquelas com os tipos B e F não-proteolíticas; cepas produtoras de toxinas C e D são do grupo III; e o grupo IV é representado por cepas do tipo $\mathrm{G}^{4}$.

O quadro clínico de botulismo, nas suas quatro categorias, é essencialmente o mesmo. Os primeiros sintomas são geralmente náuseas, vômitos, dor abdominal, diarréia e constipação. Os sinais neurológicos se instalam com manifestações de cefaléia, prejuízos visuais como diplopia, pupila dilatada, ptose palpebral, nistagmo, oftalmoplegia, disfagia, disartria, boca, garganta e língua secas, paralisia descendente, fraqueza muscular progressiva, iniciando-se da região cervical estendendo-se aos membros superiores, tórax e membros inferiores, dilatação gástrica, íleo paralisado, retenção urinária, distonia, fatiga generalizada, perda do controle muscular e comprometimento respiratório, sendo essa última a principal causa de óbitos. $\mathrm{O}$ indivíduo permanece consciente ${ }^{2-4}$.

Nos casos de botulismo alimentar, objeto deste relato, o período de incubação é variável, sendo mais comum entre 12 e $36 \mathrm{~h}^{2}$, podendo ser em menor prazo, apresentando-se em apenas $2 \mathrm{~h}$, conforme a quantidade e tipo de toxina ingerida. Pode levar a óbito em menos de $24 \mathrm{~h}$. Somente uma pequena percentagem de toxina ingerida é absorvida pela mucosa intestinal, sendo o restante eliminado nas fezes. Uma vez absorvida, a toxina é transportada via hematógena até neurônios sensíveis ${ }^{5}$.

Embora grave, é uma doença pouco diagnosticada pelos profissionais de saúde, o que geralmente leva a um tratamento tardio e ineficaz ${ }^{4}$. No Brasil, o primeiro caso de botulismo notificado à Secretaria de Vigilância em Saúde ocorreu em 2002; no entanto, desde 1999, a Coordenação de Vigilância das Doenças de Transmissão Hídrica e Alimentar realiza a vigilância dessa doença. O primeiro relato de um surto epidêmico de botulismo no Brasil aconteceu no Estado do Rio Grande do Sul, em 1958, onde nove pessoas morreram após consumir conserva de peixe caseiro ${ }^{3,6}$. Entre 1999 e 2006, foram notificados 66 casos suspeitos de botulismo, dentre os quais somente 32 foram confirmados, onde $96,8 \%$ foram de origem alimentar. 
Este relato descreve um surto familiar, ocorrido na Cidade de Fortaleza, capital do Estado do Ceará, cujo alimento suspeito foi uma torta de frango consumida pelos pacientes em sua residência.

\section{RELATO DE CASO}

As informações apresentadas foram obtidas no Centro de Assistência Toxicológica (CEATOX), do Instituto José Frota (IJF), em Fortaleza, Ceará. A investigação constou de consultas aos prontuários dos pacientes e nos registros nas agências municipais de vigilância sanitária e epidemiológica.

Segundo a Ficha de Notificação e de Atendimento do CEATOX, duas crianças do sexo feminino, irmãs, uma com 12 anos (paciente I) e outra com 10 anos (paciente II), foram atendidas no dia 25/09/2006 apresentando sintomas de intoxicação do tipo aguda. As pacientes chegaram com suspeita de envenenamento via alimento contaminado. Foram submetidas à ventilação mecânica (VM) e entubadas. Foram administrados atropina e carvão ativado. Houve suspeita de botulismo logo no primeiro dia. A mãe das crianças (paciente III) procurou ajuda médica no dia 26/09/2006, apresentando, também, sintomas de intoxicação do tipo aguda. Os sintomas e sinais apresentados por cada uma das pacientes, bem como a evolução temporal da doença estão apresentados na Tabela 1.

As pacientes foram internadas na Unidade de Terapia Intensiva (UTI). A partir de informações coletadas junto à mãe das crianças (paciente III), o alimento suspeito passou a ser uma torta de frango oferecida por uma vizinha, $24 \mathrm{~h}$ antes do aparecimento dos sintomas. Os procedimentos adotados para cada paciente foram direcionados às suas maiores necessidades, conforme descrito a seguir.

\section{Paciente I}

Teve suspenso o tratamento com carvão ativado, 24h após sua internação, para coleta de secreção gástrica, onde seria posteriormente feito o teste para botulismo. Administração imediata do soro antibotulínico. Vinte e dois dias após sua entrada no hospital, a paciente I apresentava melhora e já se comunicava com a equipe, mas continuava na UTI. Quarenta e dois dias depois do início dos sintomas a paciente recebeu alta médica, mas apresentava sequelas que não foram especificadas no prontuário.

\section{Paciente II}

Foi submetida à lavagem gástrica e análise toxicológica que demonstrou inibição da colinesterase $(14,5$ [5,0-16,5]). Foi solicitado exames para pesquisa de toxina botulínica e hemocultura, com administração de soro antibotulínico. Houve suspeita de morte cerebral três dias após a entrada no IJF. O exame de eletroencefalograma refutou a suspeita. Todavia, a criança continuava sem responder a estímulos. Segundo o prontuário, a paciente II apresentava sequelas e permaneceu em estado grave na UTI por cinco dias, quando foi a óbito.

\section{Paciente III}

O soro antibotulínico foi administrado; entretanto, a paciente continuou desorientada e em estado grave. Houve suspeita de úlcera de córnea. Treze dias após a internação apresentou melhora, apenas com dificuldade respiratória. Apresentou quadro de infecção respiratória e foi tratada. O quadro manteve-se estável e a paciente foi transferida para o leito comum. No dia 06/11/2006, a paciente recebeu alta e, segundo o prontuário, apresentava sequelas não especificadas no prontuário.

Não foram encontrados registros sobre os resultados da toxicologia relacionados às amostras clínicas das pacientes e do alimento suspeito.

TABELA 1 - Resumo sintomatológico, tempo de internação e conclusão de três casos de botulismo familiar acontecido em Fortaleza, Estado do Ceará, no ano de 2006*.

\begin{tabular}{llcl}
\hline Paciente & Quadro sintomatológico & $\begin{array}{c}\text { Duração do } \\
\text { internamento }\end{array}$ & Conclusão do caso \\
\hline I & Úlcera de córnea, insuficiência respiratória, atelectasia, febre e pupilas midriáticas & 42 dias & $\begin{array}{l}\text { Paciente liberada apresentando } \\
\text { sequelas não especificadas no prontuário }\end{array}$ \\
\hline II & $\begin{array}{l}\text { Úlcera de córnea, insuficiência respiratória, atelectasia, febre e pupilas midriáticas, } \\
\text { convulsão, broncoespasmos, mioclonia e desorientação, sendo sedada e analgesiada }\end{array}$ & 5 dias & Óbito \\
\hline III & $\begin{array}{l}\text { Tontura, fraqueza muscular progressiva, sonolência e vômitos, reflexo pupilar paradoxal, } \\
\text { miótico à luz e midriático ao abrir os olhos, sem queixas visuais. }\end{array}$ & Pl dias & $\begin{array}{l}\text { naciente liberada apresentando sequelas } \\
\text { neve desorientação, mas manteve-se consciente. }\end{array}$ \\
\hline *Informaçãos no prontuário
\end{tabular}

\section{DISCUSSÃO}

De evolução dramática, o surto familiar teve duração de quarenta e um dias em Unidade de Terapia Intensiva. O grupo do Centro de Toxicologia do Estado do Ceará mostrou-se eficiente no diagnóstico da doença, administrando o soro antibotulínico, em tempo hábil. Em uma análise da epidemiologia, relatos clínicos e dos diagnósticos laboratoriais de casos de botulismo no Brasil, no período de 2000 a 2008 , foi encontrada uma taxa de $34,2 \%$ de mortalidade entre os casos registrados. Os autores alertaram para o fato de o botulismo ser uma doença rara e, por isso, muitos médicos não estão familiarizados com os sintomas. Erros ou atrasos no diagnóstico resultam em demora na administração do soro antibotulínico e como consequência o aumento da mortalidade. A estrutura dos hospitais que receberam os pacientes com botulismo foi um fator determinante na redução da taxa de mortalidade. Foram verificadas taxas menores nos casos em que os pacientes foram atendidos em unidades hospitalares que dispunham de unidades de terapia intensiva? ${ }^{7}$.

São de responsabilidade das unidades de atendimento a coleta de informações e o acionamento do sistema de monitoramento desses surtos alimentares. Para uma investigação eficiente, é importante o preenchimento adequado e exato dos formulários e prontuários. Durante esta pesquisa, foram verificadas falhas no preenchimento dos prontuários das pacientes. A ausência de informações relevantes como detalhamento na discriminação das sequelas apresentadas 
pelas pacientes que sobreviveram e a falta de registros dos resultados dos exames laboratoriais dificultaram o estudo do surto. Segundo Oliveira cols ${ }^{8}$, é crescente, no Brasil, a importância da informação em saúde para o planejamento e gestão de recursos assim como para fundamentar decisões sobre medidas de promoção e proteção da saúde além das ações de prevenção e controle de doenças no indivíduo e nas populações. Para a eficiência do processo, é crucial a qualidade dos dados e, de forma particular, a notificação e investigação de casos e de surtos são fundamentais uma vez que permitem o conhecimento da magnitude dos agravos e a tomada de medidas preventivas pertinentes.

O botulismo é uma doença de notificação compulsória (Portaria do Ministério da Saúde $\mathrm{n}^{\circ}$ 1943, de 18 de outubro de 2001). Devido à gravidade dessa doença e o risco relacionado à ingestão da mesma fonte de alimentos contaminados, um caso de botulismo é considerado um surto exigindo notificação imediata e investigação por parte da vigilância epidemiológica local.

De acordo com o Manual do Botulismo ${ }^{10}$, o profissional de saúde responsável pelo atendimento de pacientes com suspeita da doença deve notificar a Vigilância Epidemiológica, e esta, por sua vez, deve investigar todo o histórico do paciente incluindo resultados de exames. Também fazem parte das tarefas da Vigilância Epidemiológica a investigação e o rastreamento dos alimentos consumidos dentro de um período de cinco dias, estabelecendo a procedência deste alimento, seu armazenamento e o consumo comum entre o paciente e os demais familiares. A Vigilância Sanitária deve ser acionada para realização da coleta de alimentos suspeitos, na casa dos pacientes ou estabelecimentos onde foram adquiridos, para análise toxicológica.

No caso apresentado, o procedimento foi adequado de acordo com o que preconiza o Manual do Botulismo ${ }^{6} . \mathrm{O}$ alimento (torta de frango) relacionado como possível veículo de contaminação foi recolhido para análise. Informações pertinentes sobre o alimento suspeito também não foram disponibilizadas como a procedência (doméstica ou industrial). Segundo dados da Secretaria de Vigilância em Saúde (SVS), em levantamento sobre botulismo alimentar, registrado no Brasil, no período de 1999 a 2008, 8 (21\%) casos foram envolvidos com alimentos do tipo torta de frango tanto artesanais como industriais.

Em São Paulo, aconteceram dois surtos de botulismo relacionados à ingestão de tortas de frango ${ }^{9,10}$. Nestes casos, as tortas foram produzidas em estabelecimentos comerciais e tinham palmito como um dos ingredientes do recheio. A investigação sanitária verificou falhas na preparação e armazenamento destes produtos resultando em condições propícias para a formação de toxina botulínica.
Sendo o botulismo um problema de saúde pública, e considerandose os dados apresentados, fica um alerta para os profissionais de saúde, no que tange à precisão e cuidado no preenchimento de dados relevantes para a notificação de doenças compulsórias. Além disto, são importantes o treinamento e a conscientização dos profissionais da área da saúde sobre a integração hospital-vigilância epidemiológica e vigilância sanitária para que o sistema de informação e análise da vigilância epidemiológica permita um monitoramento adequado e eficiente de surtos ocorridos no país.

\section{AGRADECIMENTOS}

Ao Centro de Assistência Toxicológica (CEATOX) do Instituto José Frota (IJF).

\section{REFERÊNCIAS}

1. Centers for Disease Control and Prevention. Botulism in the United States, 1899-1996: handbook for epidemiologists, clinicians, and laboratory workers. Atlanta: Center For Disease Control and Prevention; 1998.

2. Cardoso T, Costa M, Almeida HC, Guimarães M. Botulismo alimentar: estudo retrospectivo de cinco casos. ACTA Medica Portuguesa 2004; 17:54-58

3. Cereser ND, Costa FMR, Rosii Júnior OD, Silva DAR, Sperotto VR. Botulismo de origem alimentar. Cienc Rural 2008; 38:280-287.

4. Figueiredo MAA, Dias J, Lucena R. Considerações acerca de dois casos de botulismo ocorridos no Estado da Bahia. Rev Soc Bras Med Trop 2006; 39:289291.

5. Ferreira MCS, Domingues RMCP. Clostridium. In: Trabulsi LR, Alterthum F, editores. Microbiologia. $5^{\text {a }}$ ed. São Paulo: Atheneu; 2004. p. 400-401.

6. Secretaria de Saúde do Estado de São Paulo. Botulismo: orientações para pacientes e familiares [Internet]. [Acesso $20 \mathrm{dez} 2009$ ]. Disponível em: http://www.saude. sp.gov.br/resources/cidadao/destaques/botulismo-c/.

7. Rowlands REG, Ristori CA, Lopes GISL, Paula AMR, Sakuma H, Grigaliunas $\mathrm{R}$, et al. Botulism in Brazil, 2000-2008: epidemiology, clinical findings and laboratorial diagnosis. Rev Inst Med Trop Sao Paulo 2010; 52:183-186.

8. Oliveira MEP, Soares MRAL, Costa MCN, Mota ELA. Avaliação da completitude dos registros de febre tifóide notificados no Sinan pela Bahia. Epidemiol Serv Saude 2009; 18:219-226.

9. Eduardo MBP, Madalosso G, Paiva OR, Brito SN, Araújo EC, Bandeira CRS, et al. Botulismo tipo A e B causado por torta comercial de frango com palmito e ervilhas no Município de São Paulo, SP - Janeiro de 2007. Bol Epi Pal 2007; 38:1-7.

10. Coordenadoria de Controle de Doenças. Centro de Vigilância Epidemiológica "Prof. Alexandre Vranjac". Divisão de Doenças de Transmissão Hídrica e Alimentar. Botulismo e Torta comercial de frango com requeijão. Bol Epi Pal 2006; 27:14-19. 